\title{
Evidenz versus Politik
}

B ei vielen Gelegenheiten wird in diesem Jahr in allergologischen Zeitschriften oder im Rahmen von Kongressen an die ersten Mitteilungen über die Möglichkeit einer spezifischen Immuntherapie bei wie wir heute wissen - Immunglobulin-E-abhängigen allergischen Erkrankungen wie Rhinitis allergica oder allergischem Asthma erinnert. Eine Aufstellung wichtiger geschichtlicher Daten von Jan Gutermuth und Johannes Ring finden Sie dazu in dieser Ausgabe des Allergo Journal.

Es waren jedoch vor allem die akut gefährdenden Allergien auf Hymenopterengift, an deren Beispiel die Wirksamkeit der spezifischen Hyposensibilisierung nicht nur überzeugend demonstriert werden konnte, sondern die auch zum bevorzugten Model wurden, um die Wirksamkeit dieser Therapieform zu untersuchen. In dieser Ausgabe des Allergo Journal finden Sie die neueste Leitlinie zur Behandlung der IgE-abhängigen spezifischen Immuntherapie bei Hymenopterengiftallergie. Dieses Gebiet bleibt spannend, nicht zuletzt auch durch die Herausforderungen, vor die uns infolge von Klimaveränderungen neu oder verstärkt auftretende Wespenarten stellen (Mauss V. Bionomie und Abwehrverhalten der in Deutschland vorkommenden allergologisch bedeutsamen Bienen und Faltenwespen. Hautarzt 2008; 59: 184-93).

Gegenwärtig besteht eine Diskrepanz zwischen der Leistungsfähigkeit der spezifischen Immuntherapie und deren tatsächlicher Ausnutzung. Die Wirksamkeit und die Handhabbarkeit der Therapieform verbessert sich stetig durch Neuentwicklungen und Modifikationen der Applikationsform von

„Die Hyposensibilisierung ist integraler Bestandteil eines

Therapiemanagements bei IgEabhängigen allergischen Reaktionen; eine Einordnung z. B. als IGeL-Leistung wäre abwegig. “ der Toleranzinduktion gezielt modifizierter Antigene. Auch neue Kombinationen mit Medikamenten zur Optimierung der Wirksamkeit lassen sich denken (Akdis M, Akdis CA. Therapeutic manipulation of immune tolerance in allergic disease. Nat Rev Drug Discov 2009; 8: 645-60 / Wedi B, Ruëff F. Pharmakoprophylaxe und Begleitmedikation bei spezifischer Immuntherapie. Hautarzt, im Druck). Trotzdem beobachten wir als Folge einer gesundheitspolitischen Fehlsteuerung eine deutliche Reduktion der Patienten, bei denen eine spezifische Immuntherapie neu begonnen wird.

$\mathrm{Da}$ wir heute gute Evidenzen nicht nur für einen kurativen Effekt der Hyposensibilisierung über den eigentlichen Behandlungszeitraum hinaus haben, sondern auch einen prophylaktischen Schutz vor dem gefürchte-

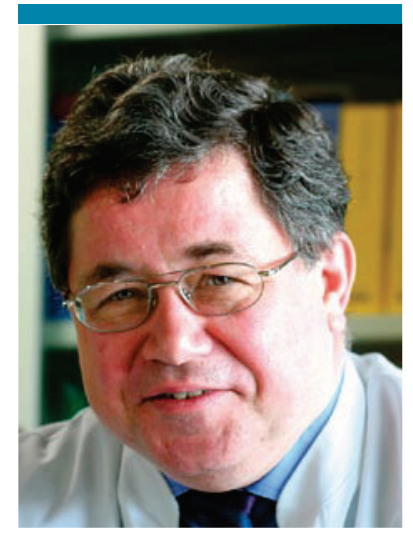

Prof. Dr. Hans F. Merk, Hautklinik der Medizinischen Fakultät, Universitätsklinikum der RWTH Aachen

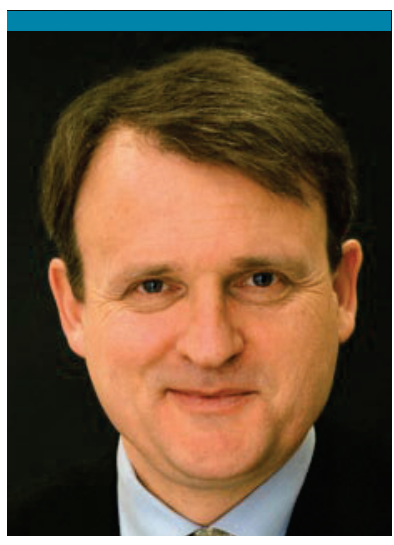

Prof. Dr. Thilo Jakob, Allergieabteilung \& Forschergruppe Allergologie, UniversitätsHautklinik Freiburg ten „Etagenwechsel“, ist eine rasche Korrektur dieser Fehlsteuerung geboten. Ein mehrjähriges Abwarten der Ergebnisse von Selektivvertragserprobungen in Bayern bis zu bundesweiten Korrekturen wäre eine verhängnisvolle Verzögerung, für die keine Zeit mehr bleibt. Auch sind die beanspruchten Effekte dieser Therapieform für viele angebotene Präparate - unabhängig von der Applikationsform SCIT oder SLIT - durch Studien belegt, die nach modernen Kriterien der klinischen Pharmakologie durchgeführt wurden. Daher ist die Hyposensibilisierung integraler Bestandteil eines Therapiemanagements bei IgE-abhängigen allergischen Reaktionen; eine Einordnung z. B. als IGeL-Leistung wäre abwegig. In politischen Entscheidungsgremien sollte man nicht übersehen, dass die von diesen Krankheiten betroffenen Patienten etwa das 5\%-Quorum bei Bundestagswahlen mehrfach übertreffen.

Umso wichtiger ist die Teilnahme aller an der Allergie im deutschsprachigen Raum Interessierten am 6. Deutschen Allergiekongress vom 8. bis 10. September dieses Jahres in Wiesbaden. Denn dort werden auch berufspolitische Themen zur spezifischen Immuntherapie diskutiert. Zudem werden in einem von Prof. Dr. Ludger Klimek und Prof. Dr. Wolfgang Schlenter hervorragend vorbereiteten organisatorischen Rahmen auch viele andere Bereiche unseres spannenden Fachs präsentiert.

Wir freuen uns darauf, Sie beim Kongress zu sehen und gemeinsam anregende Tage zu erleben.

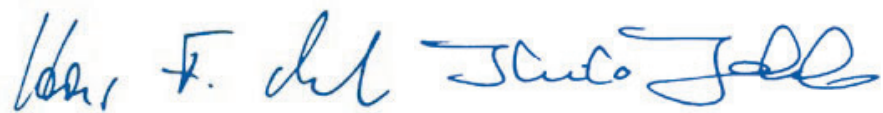

Prof. Dr. Hans F. Merk ～Prof. Dr. Thilo Jakob 